\title{
Combining Ability Studies for Various Horticultural Traits in Garden Pea (Pisum sativum L.)
}

\author{
Manoj Kumar ${ }^{1}$, R. K. Gupta ${ }^{1}$, Sanjeev Kumar ${ }^{1 *}$, R. K. Samnotra ${ }^{1}$, \\ Anil Bhushan ${ }^{1}$ and Tuhina Dey ${ }^{2}$ \\ ${ }^{1}$ Division of Vegetable Science and Floriculture, ${ }^{2}$ Division of Plant Breeding and Genetics, \\ SKUAST - J, J\&K, India \\ *Corresponding author
}

\section{A B S T R A C T}

\section{Keywords}

Line $\mathrm{x}$ tester, Pea, Combining ability, Grain Yield, Protein content, GCA and SCA

\section{Article Info}

Accepted:

10 November 2020

Available Online:

10 December 2020
Combining ability effects were estimated for different characters in a line $\mathrm{x}$ tester crossing programme comprising 36 hybrids produced by crossing 12 lines and 3 testers. Parents and hybrids differed significantly for gca and sca effects, respectively. Among the lines, Palam Triloki, Vivek Matar-10, Palam Priya, Azad Pea-3, Arka Karthik and Arka Ajit and among the testers P-89 and VL-Ageti Matar-7 were found to be the best general combiners. Six crosscombiners viz., Palam Priya x VL-Ageti Matar-7, Palam Priya x Azad Pea-1, Arka Ajit x P-89, Arka Ajit x VL-Ageti Matar-7, Arka Ajit x Azad Pea-1 and Azad Pea-3 x P-89 were found to be the best specific crosses for yield per plant and other related traits. Result showed that non additive gene action is an integral component of the genetic architecture of different traits in the material used in pea.

\section{Introduction}

Garden pea (Pisum sativum L.) a diploid $(2 \mathrm{n}=2 \mathrm{x}=14)$ cultivated species of genus Pisum, family Leguminosae, is native of region between Mediterranean and Near East. It is grown both for fresh market and for food processing industry across the world. India rank $2^{\text {nd }}$ in area and production of green peas after China and rank $5^{\text {th }}$ in terms of yield in world. In India, it is grown on an area of 540.5 thousand hectares with an annual production of 5422.1 thousand MT with a productivity level of $10.0 \mathrm{MT} / \mathrm{ha}$ (Anonymous 2017-18). In Jammu \& Kashmir, it covers an area of 2.38 thousand hectare as winter and off season crop with an annual production of 30.82 thousand MT. and productivity level of $12.95 \mathrm{MT} / \mathrm{ha}$ (Anonymous 2017-18). Hybridization is one of the important breeding methods for breaking yield barriers. It offers for great possibilities in crop improvement than any other breeding method and is the only effective means of combining together the desirable horticultural traits of two or more 
varieties, by the concept of combining ability, it refers to capacity of genotype to transmit superior performance to its progeny (Sprague and Tatum, 1942).

\section{Materials and Methods}

Experimental material comprised of twelve lines and three testers (Table 1). The testers were crossed with each line and thus $36 \mathrm{~F}_{1}$ 's were produced (Table 2). The 15 parents along with $36 \mathrm{~F}_{1}, \mathrm{~s}$ were grown in a Randomized Block Design with three replications at the Experimental Farm, Division of Vegetable Science \& Floriculture, Faculty of Agriculture, Sher-e Kashmir University of Agricultural Sciences and Technology Jammu, Main Campus, Chatha, Jammu (J\&K). The distance between the plants was maintained at $10 \mathrm{~cm}$ while the rows were spaced $45 \mathrm{~cm}$ apart. The standard plant protections and other cultural practices were followed to maintain uniform experimental conditions. Five plants of each entry in each replication were randomly selected (excluding boarder rows) for recording the observations on various characters namely plant height $(\mathrm{cm})$, days to 50 per cent flowering, node at which first flower appears, inter nodal distance $(\mathrm{cm})$, number of pods per node, days to first picking, pod length $(\mathrm{cm})$, pod width $(\mathrm{cm})$, number of pods per plant, number of seed per pod, number of primary branches per plant, shelling per cent age, total sugar content $(\%)$, crude protein $(\%)$, total soluble solids $\left(\mathrm{B}^{0}\right)$, pod yield per plant (g) and pod yield per hectare (q). The analysis of general and specific combining abilities for the above traits were calculated as per the model suggested by Kempthorne (1957).

\section{Results and Discussion}

The analysis of variance showed significant differences among the crosses for all the traits except for ascorbic acid content (Table 3). Mean squares due to crosses were further portioned into lines, testers and line $\mathrm{x}$ testers interactions. Mean square due to lines were found significant for traits namely plant height, days to 50 per cent flowering, ascorbic acid content, total sugar content, crude protein, total soluble solids, pod yield per plant and pod yield per hectare. Mean squares due testers were found to be significant for eleven traits namely plant height, days to 50 per cent flowering, intermodal distance, days to first picking, pod length, number of seed per pod, shelling percentage, total sugar content, crude protein, pod yield per plant and pod yield per hectare. Line $\mathrm{x}$ tester interactions were found significant for all the traits under study except days to first picking.

\section{Estimates of general combining ability effects}

The estimates of gca effects (Table 4) showed that the parental line Palam Triloki was found to be the best general combiner for pod yield per hectare, pod yield per plant, crude protein and total soluble solids; Vivek Matar-10 for pod yield per hectare, plant height, days to $50 \%$ flowering, number of pods per node, days to first picking, total sugar content; Palam Priya for pod yield per hectare, pod yield per plant, plant height, number of pods per plant, number of primary branches per plant, shelling percentage, crude protein; Azad Pea-3 for pod yield per hectare, pod yield per plant, node at which first flower appears, pod width, number of seeds per pod, crude protein; Arka Karthik for plant height, node at which first flower appears, number of pods per node, pod length, number of pods per plant, number of seeds per pod, number of primary branches per plant, total sugar content, crude protein; Arka Ajit for pod length, number of primary branches per plant, total sugar content; Arka Apoorva for days to $50 \%$ flowering, days to first picking, pod 
length, number of seeds per pod, number of primary branches per plant, shelling percentage, ascorbic acid content, crude protein; Arka Priya for days to $50 \%$ flowering, node at which first flower appears, inter nodal distance, days to first picking, shelling percentage, total soluble solids; Arkel for number of pods per node, days to first picking, pod length, number of pods per plant, number of seeds per pod, total sugar content; Vivek Matar-11 for plant height, node at which first flower appears, number of pods per node, number of pods per plant; Matar Ageta-7 for days to $50 \%$ flowering, days to first picking, pod length, number of seeds per pod, total soluble solids; Mithi Phali for plant height, number of pods per node, total soluble solids.

Table.1 List of parents (Males and Females)

\begin{tabular}{|c|c|}
\hline S. No. & Genotype (s) \\
\hline Female parents & Palam Triloki \\
\hline $\mathbf{1}$ & Palam Priya \\
\hline $\mathbf{2}$ & Arka Karthik \\
\hline $\mathbf{3}$ & Arka Ajit \\
\hline $\mathbf{4}$ & Arka Apoorva \\
\hline $\mathbf{5}$ & Arka Priya \\
\hline $\mathbf{6}$ & Azad Pea-3 \\
\hline $\mathbf{7}$ & Arkel \\
\hline $\mathbf{9}$ & Vivek Matar-10 \\
\hline 10 & Vivek Matar-11 \\
\hline 11 & Matar Ageta-7 \\
\hline 12 & Mithi Phali \\
\hline Male parents & \\
\hline 1 & P-89 \\
\hline 2 & Azad Pea-1 \\
\hline 3 & VL-Ageti Matar-7 \\
\hline
\end{tabular}

Table. 2 Hybrids $\left(\mathrm{F}_{1}{ }^{\prime} \mathrm{s}\right)$ developed by crossing 12 lines and 3 testers

\begin{tabular}{|c|c|c|c|}
\hline S. No. & Hybrids & S. No. & Hybrids \\
\hline 1 & Palam Priya x P-89 & 19 & Vivek Matar-11 x P-89 \\
\hline 2 & . Palam Priya x VL-Ageti Matar-7 & 20 & Vivek Matar-11 x VL-Ageti Matar-7 \\
\hline 3 & Palam Priya x Azad Pea-1 & 21 & Vivek Matar-11 x Azad Pea-1 \\
\hline 4 & Arka Ajit x P-89 & 22 & Arka Priya x P-89 \\
\hline 5 & Arka Ajit x VL-Ageti Matar-7 & 23 & Arka Priya x VL-Ageti Matar-7 \\
\hline 6 & Arka Ajit x Azad Pea-1 & 24 & Arka Priya x Azad Pea-1 \\
\hline 7 & Azad Pea-3 x P-89 & 25 & Mithi Phali x P-89 \\
\hline 8 & Azad Pea-3 x VL-Ageti Matar-7 & 26 & Mithi Phali x VL-Ageti Matar-7 \\
\hline 9 & Azad Pea-3 x Azad Pea-1 & 27 & Mithi Phali x Azad Pea-1 \\
\hline 10 & Arka Apoorva x P-89 & 28 & Matar Ageta- 7 x P-89 \\
\hline 11 & Arka Apoorva x VL-Ageti Matar-7 & 29 & Matar Ageta- 7 x VL-Ageti Matar-7 \\
\hline 12 & Arka Apoorva x Azad Pea-1 & 30 & Matar Ageta- 7 x Azad Pea-1 \\
\hline 13 & Arka Karthik x P-89 & 31 & Vivek Matar-10 x P-89 \\
\hline 14 & Arka Karthik x VL-Ageti Matar-7 & 32 & Vivek Matar-10 x VL-Ageti Matar-7 \\
\hline 15 & Arka Karthik x Azad Pea-1 & 33 & Vivek Matar-10 x Azad Pea-1 \\
\hline 16 & Palam Triloki x P-89 & 34 & Arkel x P-89 \\
\hline 17 & Palam Triloki x VL-Ageti Matar-7 & 35 & Arkel x VL-Ageti Matar-7 \\
\hline 18 & Palam Triloki x Azad Pea-1 & 36 & Arkel x Azad Pea-1 \\
\hline
\end{tabular}


Table.3 Analysis of variance for combining ability for various traits in peas (Pisum sativum L.)

\begin{tabular}{|c|c|c|c|c|c|c|c|c|c|c|}
\hline $\begin{array}{l}\text { Source of } \\
\text { variation }\end{array}$ & d.f. & $\begin{array}{c}\text { Plant } \\
\text { Height }(\mathbf{c m})\end{array}$ & $\begin{array}{c}\text { Days to } \\
50 \% \\
\text { flowering }\end{array}$ & $\begin{array}{c}\text { Node at } \\
\text { which first } \\
\text { flower } \\
\text { appears }\end{array}$ & $\begin{array}{c}\text { Inter } \\
\text { nodal } \\
\text { distance } \\
(\mathrm{cm})\end{array}$ & $\begin{array}{c}\text { Number } \\
\text { of pods } \\
\text { per node }\end{array}$ & $\begin{array}{c}\text { Days to } \\
\text { first } \\
\text { picking }\end{array}$ & $\begin{array}{l}\text { Pod length } \\
\quad(\mathrm{cm})\end{array}$ & $\begin{array}{l}\text { Pod width } \\
\quad(\mathrm{cm})\end{array}$ & $\begin{array}{c}\text { Number of pods } \\
\text { per plant }\end{array}$ \\
\hline Replicates & 2.00 & 101.15 & 1.36 & 15.91 & 4.81 & 0.01 & 0.56 & 0.04 & 0.03 & 213.87 \\
\hline Crosses & 35.00 & $1152.35^{*}$ & $313.69 *$ & $4.09 *$ & $1.21 *$ & $0.02 *$ & $330.87 *$ & $2.11 *$ & $4.25^{*}$ & $821.02 *$ \\
\hline Lines & 11.00 & $2373.78 *$ & $430.16 *$ & 4.42 & 1.39 & 0.03 & 413.01 & 1.65 & 4.34 & 870.42 \\
\hline Testers & 2.00 & $1901.41 *$ & $1365.75 * *$ & 1.46 & $3.90^{*}$ & 0.02 & $1277.06^{* *}$ & $14.58 * *$ & 4.47 & 84.33 \\
\hline $\begin{array}{l}\text { Lines } \mathrm{x} \\
\text { Tester }\end{array}$ & 22.00 & $23.75^{* *}$ & $159.82 *$ & $4.16^{* *}$ & $0.88 * *$ & $0.02 * *$ & 203.79 & $1.21 *$ & $4.19 * *$ & $863.30 * *$ \\
\hline Error & 70.00 & 23.75 & 0.36 & 0.23 & 0.40 & 0.01 & 1.73 & 0.12 & 0.02 & 38.69 \\
\hline
\end{tabular}

\begin{tabular}{|c|c|c|c|c|c|c|c|c|c|c|}
\hline $\begin{array}{l}\text { Source of } \\
\text { variation }\end{array}$ & d.f. & $\begin{array}{l}\text { Number } \\
\text { of seed } \\
\text { per pod }\end{array}$ & $\begin{array}{l}\text { Number of } \\
\text { primary } \\
\text { branches } \\
\text { per plant }\end{array}$ & $\begin{array}{c}\text { Shelling } \\
\text { percentage } \\
(\%)\end{array}$ & $\begin{array}{c}\text { Ascorbic } \\
\text { Acid } \\
\text { content } \\
\text { (mg/100g) }\end{array}$ & $\begin{array}{c}\text { Total } \\
\text { Sugar } \\
\text { Content } \\
(\%)\end{array}$ & $\begin{array}{c}\text { Crude } \\
\text { Protein } \\
(\%)\end{array}$ & $\begin{array}{c}\text { Total } \\
\text { Soluble } \\
\text { Solids } \\
\left(\text { Brix }=\mathbf{B}^{\mathbf{0}} \text { ) }\right.\end{array}$ & $\begin{array}{l}\text { Pod yield } \\
\text { per plant } \\
\text { (g) }\end{array}$ & $\begin{array}{l}\text { Pod yield } \\
\text { per hectare } \\
\text { (q) }\end{array}$ \\
\hline Replicates & 2.00 & 0.11 & 0.16 & 8.12 & 0.00 & 0.99 & 0.63 & 0.21 & 19.89 & 98.04 \\
\hline Crosses & 35.00 & $2.73 *$ & $224 *$ & $9.01 *$ & 0.29 & $15.82 *$ & $13.50^{*}$ & $4.23 *$ & $452.89 *$ & $2237.06^{*}$ \\
\hline Lines & 11.00 & 2.80 & 2.96 & 8.40 & $0.53 *$ & $48.23^{*}$ & $13.06^{*}$ & $8.18 *$ & $466.35^{*}$ & $2305.47^{*}$ \\
\hline Testers & 2.00 & $9.40 *$ & 1.52 & $42.93 * *$ & 0.20 & $4.96 * *$ & $133.30 *$ & 5.72 & $4983.05^{*}$ & $24602.94^{*}$ \\
\hline Lines $\mathrm{x}$ testers & 22.00 & $2.09 * *$ & $1.95^{* *}$ & $6.24 *$ & $0.18 * *$ & $0.61 *$ & $2.82 * *$ & $2.11 * *$ & $34.33 * *$ & $169.59 * *$ \\
\hline Error & 70.00 & 0.04 & 0.04 & 0.78 & 0.02 & 0.05 & 0.06 & 0.05 & 5.22 & 25.87 \\
\hline
\end{tabular}

$*$ Significant at $5 \%$ level of significance $* *$ Significant at $1 \%$ level of significance 
Table.4 Estimates of General Combining Ability (GCA) effects of lines for various traits in peas (Pisum sativum L.)

\begin{tabular}{|c|c|c|c|c|c|c|c|c|c|}
\hline Female Parent & $\begin{array}{l}\text { Plant } \\
\text { Height } \\
\text { (cm) }\end{array}$ & $\begin{array}{c}\text { Days to } \\
50 \% \\
\text { flowering }\end{array}$ & $\begin{array}{c}\text { Node at } \\
\text { which first } \\
\text { flower } \\
\text { appears }\end{array}$ & $\begin{array}{c}\text { Inter } \\
\text { nodal } \\
\text { distance } \\
(\mathbf{c m})\end{array}$ & $\begin{array}{c}\text { Number } \\
\text { of pods } \\
\text { per node }\end{array}$ & $\begin{array}{c}\text { Days to } \\
\text { first } \\
\text { picking }\end{array}$ & $\begin{array}{l}\text { Pod length } \\
\quad(\mathrm{cm})\end{array}$ & $\begin{array}{l}\text { Pod width } \\
\quad(\mathrm{cm})\end{array}$ & $\begin{array}{c}\text { Number } \\
\text { of pods } \\
\text { per plant }\end{array}$ \\
\hline Palam Triloki & $-22.09 *$ & $4.92 * *$ & $0.85^{* *}$ & -0.39 & $-0.05^{*}$ & $4.96 * *$ & $-0.49 * *$ & $-0.27 * *$ & $-14.64 * *$ \\
\hline Palam Priya & $6.62 * *$ & $3.92 *$ & -0.12 & 0.10 & 0.02 & $3.29 * *$ & -0.21 & $-0.29 * *$ & $10.79 * *$ \\
\hline Arkel & 2.42 & $-2.08 *$ & -0.28 & 0.13 & $0.05^{* *}$ & $-1.21 *$ & $0.32 * *$ & $-0.20 * *$ & $14.48 * *$ \\
\hline Arka Karthik & $22.07 *$ & $9.42 *$ & $-0.85 * *$ & -0.22 & $0.05 * *$ & $10.29 * *$ & $0.42 * *$ & 0.01 & $10.21 *$ \\
\hline Arka Ajit & $-8.41 * *$ & $8.92 * *$ & -0.25 & 0.26 & $-0.08 *$ & $7.12 *$ & $0.73 * *$ & $-0.23 * *$ & -3.327 \\
\hline Arka Apoorva & 0.28 & $-15.58 * *$ & $0.61 *$ & 0.03 & $-0.08 * *$ & $-15.21 *$ & $0.32 * *$ & $-0.12 * *$ & $-5.42 * *$ \\
\hline Arka Priya & $-19.30 * *$ & $-2.92 *$ & $-0.32 * *$ & $-0.71 * *$ & $-0.05^{*}$ & $-2.54 * *$ & $-0.74 * *$ & $-0.18 * *$ & $-11.59 * *$ \\
\hline Azad Pea-3 & $-17.99 * *$ & $1.08 * *$ & $-1.14^{*}$ & 0.22 & -0.01 & $1.62 * *$ & -0.17 & $2.19 * *$ & $-7.72 * *$ \\
\hline Vivek Matar-10 & $10.47 * *$ & $-2.25 * *$ & $0.57 * *$ & 0.11 & $0.05^{* *}$ & $-2.37 * *$ & 010 & $-0.13 * *$ & $-5.52 * *$ \\
\hline Vivek Matar-1 & $27.97^{*}$ & $3.75^{* *}$ & $-0.50^{*}$ & $0.87 * *$ & $0.05 * *$ & $3.96 * *$ & -0.15 & $-0.23 * *$ & $12.41 * *$ \\
\hline Matar Ageta-7 & $-11.40 * *$ & $-4.42 * *$ & 0.26 & -0.22 & -0.01 & $-5.37 * *$ & $0.25^{*}$ & $-0.24 * *$ & 0.51 \\
\hline Mithi Phali & $9.36 * *$ & -4.75 & $1.17 * *$ & -0.18 & $0.05 * *$ & -4.54 & $-0.39 * *$ & -0.31 & -0.19 \\
\hline SE $(g i)+$ & 1.65 & 0.22 & 0.19 & 0.22 & 0.02 & 0.50 & 0.11 & 0.01 & 1.90 \\
\hline SE(gi-gj)+ & 2.33 & 0.31 & 0.27 & 0.31 & 0.03 & 0.71 & 0.15 & 0.01 & 2.67 \\
\hline$C D(p=0.05)$ & 3.29 & 0.44 & 0.3 & 0.44 & 0.04 & 1.00 & 0.21 & 0.01 & 3.77 \\
\hline$C D(p=0.01)$ & 4.37 & 0.59 & 0.51 & 0.58 & 0.05 & 1.33 & 0.28 & 0.01 & 5.01 \\
\hline
\end{tabular}

$*$ Significant at $5 \%$ level of significance **Significant at $1 \%$ level of significance 
Table.4 Estimates of General Combining Ability (GCA) effects of lines for various traits in peas (Pisum sativum L.) contd....

\begin{tabular}{|c|c|c|c|c|c|c|c|c|c|}
\hline Female Parent & $\begin{array}{c}\text { Number } \\
\text { of seed } \\
\text { per pod }\end{array}$ & $\begin{array}{c}\text { Number } \\
\text { of } \\
\text { primary } \\
\text { branches } \\
\text { per plant }\end{array}$ & $\begin{array}{c}\text { Shelling } \\
\text { percentage } \\
(\%)\end{array}$ & $\begin{array}{l}\text { Ascorbic } \\
\text { Acid } \\
\text { content } \\
(\mathbf{m g} / \mathbf{1 0 0 g})\end{array}$ & $\begin{array}{c}\text { Total } \\
\text { Sugar } \\
\text { Content } \\
(\%)\end{array}$ & $\begin{array}{c}\text { Crude } \\
\text { Protein } \\
(\%)\end{array}$ & $\begin{array}{c}\text { Total } \\
\text { Soluble } \\
\text { Solids } \\
\left(\mathbf{B r i x}=\mathbf{B}^{0}\right)\end{array}$ & $\begin{array}{c}\text { Pod yield } \\
\text { per plant } \\
\text { (g) }\end{array}$ & $\begin{array}{l}\text { Pod yield } \\
\text { per } \\
\text { hectare } \\
\text { (q) }\end{array}$ \\
\hline Palam Triloki & $-1.36 * *$ & $-0.14^{*}$ & 0.10 & $0.55^{* *}$ & $-1.24 * *$ & $0.39 * *$ & $1.24 * *$ & $17.82 * *$ & $39.60 * *$ \\
\hline Palam Priya & $-0.56^{* *}$ & $0.43 * *$ & $0.67 *$ & $0.10 *$ & $-0.95 * *$ & $1.17 * *$ & $-1.73 * *$ & $7.81 * *$ & $17.42 * *$ \\
\hline Arkel & $0.71 * *$ & 0.09 & $-0.93 * *$ & 0.01 & $0.26 * *$ & $-0.84 * *$ & -0.10 & 1.25 & 2.77 \\
\hline Arka Karthik & $0.14^{*}$ & $0.23 * *$ & $-0.93 * *$ & -0.05 & $0.35 * *$ & $1.16^{* *}$ & $-1.41 * *$ & 0.34 & 0.75 \\
\hline Arka Ajit & 0.07 & $0.83 * *$ & 0.17 & $-0.26 * *$ & $-1.14 * *$ & 0.09 & $0.59 * *$ & -0.11 & -0.24 \\
\hline Arka Apoorva & $0.54 * *$ & $0.16^{*}$ & $1.14 * *$ & $0.34 * *$ & $-0.80 * *$ & $1.45^{* *}$ & $0.20 * *$ & $-5.71 * *$ & $-12.69 * *$ \\
\hline Arka Priya & $-0.26 * *$ & $-0.24 * *$ & $0.67 *$ & 0.01 & $-0.60 * *$ & $-0.23 * *$ & $0.61 * *$ & $-1.78^{*}$ & $-3.96 *$ \\
\hline Azad Pea-3 & $0.51 * *$ & $-0.24 * *$ & 0.30 & -0.01 & $-0.58 * *$ & $1.71 * *$ & $0.21 * *$ & $2.46^{* *}$ & $5.56 * *$ \\
\hline Vivek Matar-10 & $-0.23 * *$ & $-0.21 * *$ & $-1.10 * *$ & $-0.26 * *$ & $7.17 * *$ & $-1.97 * *$ & $0.29 * *$ & $-8.64 * *$ & $19.20 * *$ \\
\hline Vivek Matar-11 & $014 *$ & $-0.41 * *$ & $-1.464 * *$ & $-0.19 * *$ & $-1.19 * *$ & $-1.59 * *$ & $-1.25^{* *}$ & $-5.96 * *$ & $-13.26 * *$ \\
\hline Matar Ageta-7 & $0.17 * *$ & $0.93 * *$ & $1.67 * *$ & $-0.17 * *$ & $-0.78 * *$ & $-0.57 * *$ & $0.92 * *$ & $-1.55 * *$ & -3.45 \\
\hline Mithi Phali & $0.14 *$ & -0.61 & -0.20 & -0.09 & -0.49 & $-0.76 * *$ & $0.42 * *$ & -5.94 & $-13.20 * *$ \\
\hline SE(gi)+ & 0.06 & 0.07 & 0.30 & 0.05 & 0.08 & 0.08 & 0.07 & 0.82 & 1.83 \\
\hline SE(gi-gj)+ & 0.09 & 0.09 & 0.43 & 0.07 & 0.11 & 0.12 & 0.10 & 1.16 & 2.59 \\
\hline$C D(p=0.05)$ & 0.13 & 0.13 & 0.60 & 0.10 & 0.16 & 0.17 & 0.14 & 1.64 & 3.66 \\
\hline $\mathrm{CD}(\mathrm{p}=0.01)$ & 0.17 & 0.17 & 0.80 & 0.13 & 0.21 & 0.22 & 0.19 & 2.18 & 4.85 \\
\hline
\end{tabular}

$*$ Significant at $5 \%$ level of significance **Significant at $1 \%$ level of significance 
Table.5 Estimates of Specific Combining Ability effects for various traits in peas (Pisum sativum L.)

\begin{tabular}{|c|c|c|c|c|c|c|c|c|c|}
\hline Hybrids & $\begin{array}{l}\text { Plant Height } \\
(\mathbf{c m})\end{array}$ & $\begin{array}{c}\text { Days to } 50 \% \\
\text { flowering }\end{array}$ & $\begin{array}{l}\text { Node at which first } \\
\text { flower appears }\end{array}$ & $\begin{array}{c}\text { Inter nodal distance } \\
(\mathbf{c m})\end{array}$ & $\begin{array}{c}\text { Number of } \\
\text { pods per node }\end{array}$ & $\begin{array}{l}\text { Days to first } \\
\text { picking }\end{array}$ & $\begin{array}{l}\text { Pod length } \\
\text { (cm) }\end{array}$ & $\begin{array}{l}\text { Pod width } \\
\text { (cm) }\end{array}$ & $\begin{array}{c}\text { Number of pods } \\
\text { per plant }\end{array}$ \\
\hline Palam Priya x P-89 & -0.34 & $-2.25 * *$ & 0.27 & -0.52 & $-0.08 *$ & $-4.29 * *$ & $-0.82 * *$ & $0.19 * *$ & -2.38 \\
\hline Palam Priya x VL-Ageti Matar-7 & -0.97 & $4.75^{* *}$ & $1.51 * *$ & -0.13 & $0.07 *$ & $6.33 * *$ & $0.73 * *$ & $-0.38^{* *}$ & -4.57 \\
\hline Palam Priya x Azad Pea-1 & 1.31 & $-2.50 * *$ & $-0.78^{* *}$ & 0.65 & 0.01 & $-2.04 *$ & 0.09 & $0.19 * *$ & $6.95 *$ \\
\hline Arka Ajit x P-89 & 4.30 & $-6.75 * *$ & -0.10 & 0.02 & 0.06 & $-8.12^{* *}$ & -0.35 & $0.31 * *$ & 4.07 \\
\hline Arka Ajit x VL-Ageti Matar-7 & $5.91^{*}$ & $5.75^{* *}$ & 0.09 & 0.56 & 0.01 & $6.00 * *$ & 0.10 & $-0.39 * *$ & 2.47 \\
\hline Arka Ajit x Azad Pea-1 & $-10.21 * *$ & $1.00^{*}$ & 0.01 & -0.58 & -0.06 & $2.12 *$ & 0.25 & $0.07 * *$ & -6.53 \\
\hline Azad Pea-3 x P-89 & -6.52 & 0.25 & $-0.89 * *$ & 0.46 & 0.02 & $4.87 * *$ & 0.12 & $0.17 * *$ & $-10.06 * *$ \\
\hline Azad Pea-3 x VL-Ageti Matar-7 & -2.21 & $-9.25 * *$ & -0.47 & -0.10 & -0.03 & $-10.50 * *$ & -0.18 & $-0.27 * *$ & $-16.39 * *$ \\
\hline Azad Pea-3 x Azad Pea-1 & $8.73 * *$ & $9.00 * *$ & $1.36^{* * *}$ & -0.36 & 0.01 & $5.62 * *$ & 0.05 & $0.10^{* * *}$ & $26.45^{* *}$ \\
\hline Arka Apoorva x P-89 & $-9.06 * *$ & -0.75 & 0.35 & 0.07 & 0.02 & -0.12 & $0.39 *$ & $0.05 * *$ & $12.31 * *$ \\
\hline Arka Apoorva x VL-Ageti Matar-7 & $6.45 *$ & $6.75 * *$ & -0.09 & 0.53 & -0.03 & $7.00 * *$ & $0.42 *$ & $-0.45^{* *}$ & $14.27 * *$ \\
\hline Arka Apoorva x Azad Pea-1 & 2.61 & $-6.00 * *$ & -0.26 & -0.60 & 0.01 & $-6.87 * *$ & $-0.81^{* *}$ & $0.40 * *$ & $-26.59 * *$ \\
\hline Arka Karthik x P-89 & 16.99 ** & $-2.25 * *$ & -0.44 & -0.67 & -0.04 & -0.96 & $0.61^{* *}$ & $0.28 * *$ & -3.94 \\
\hline Arka Karthik x VL-Ageti Matar-7 & $-7.95 * *$ & $6.75 * *$ & $-0.86^{*}$ & 0.09 & $0.11 * *$ & $5.17 * *$ & -0.31 & $-0.41 * *$ & $9.31 * *$ \\
\hline Arka Karthik x Azad Pea-1 & $-9.04 * *$ & $-4.50 * *$ & $1.29 * *$ & 0.58 & -0.06 & $-4.21 * *$ & -0.30 & $0.13^{* *}$ & -5.37 \\
\hline Palam Triloki x P-89 & $9.03 * *$ & $8.75 * *$ & $0.87 *$ & -0.02 & $0.16^{* *}$ & $9.87 * *$ & 0.22 & $0.45^{* *}$ & $9.25 * *$ \\
\hline Palam Triloki x VL-Ageti Matar-7 & $18.03^{* *}$ & $2.75 * *$ & $-1.45^{* *}$ & 0.16 & $-0.09 * *$ & $3.50 * *$ & $-0.58 * *$ & $-0.48^{* * *}$ & -5.99 \\
\hline Palam Triloki x Azad Pea-1 & $-27.06^{* *}$ & $-11.50 * *$ & 0.58 & -0.14 & -0.06 & $-13.37 * *$ & 0.35 & $0.03 * *$ & -3.25 \\
\hline Vivek Matar-11 x P-89 & -2.82 & -0.42 & $1.70^{* * *}$ & -0.35 & 0.02 & $-1.79^{*}$ & $0.82 * *$ & $0.34^{* * *}$ & $-14.88^{* *}$ \\
\hline Vivek Matar-11 x VL-Ageti Matar-7 & 0.71 & $-8.42 * *$ & $-0.75^{*}$ & -0.20 & -0.03 & $-9.67 * *$ & -0.25 & $-0.43^{* *}$ & $10.87 * *$ \\
\hline Vivek Matar-11 x Azad Pea-1 & 2.11 & $8.83 * *$ & $-0.95^{* *}$ & 0.55 & 0.01 & $11.46^{* *}$ & $-0.57 * *$ & $0.08 * *$ & 4.01 \\
\hline Arka Priya x P-89 & 0.61 & $-3.4 * *$ & 0.42 & 0.50 & $-0.11^{* *}$ & $-4.46^{* * *}$ & -0.16 & $-2.39 * *$ & -0.55 \\
\hline Arka Priyax VL-Ageti Matar-7 & -1.92 & $2.58 * *$ & -0.21 & -0.34 & 0.04 & $3.17 * *$ & $0.46^{*}$ & $4.29 * *$ & 2.11 \\
\hline Arka Priya x Azad Pea-1 & 1.31 & $0.83^{*}$ & -0.21 & -0.16 & $0.07 *$ & 1.29 & -0.29 & $-1.90^{* * *}$ & -1.55 \\
\hline Mithi Phali x P-89 & $-17.98 * *$ & 0.42 & -0.39 & -0.32 & 0.02 & -0.46 & $0.89 * *$ & $0.06^{* * *}$ & 1.05 \\
\hline Mithi Phali x VL-Ageti Matar-7 & $6.62 *$ & $-6.08^{* *}$ & 0.48 & 0.49 & -0.03 & $-5.33^{* *}$ & $-0.39^{*}$ & $-0.19^{* *}$ & 3.51 \\
\hline Mithi Phali x Azad Pea-1 & $11.35^{* *}$ & $5.67 * *$ & -0.09 & -0.17 & 0.01 & $5.79 * *$ & $-0.45^{*}$ & $0.13^{* *}$ & -4.55 \\
\hline Matar Ageta- 7 x P-89 & 0.52 & $5.92 * *$ & $-1.32 * *$ & $1.00^{*}$ & 0.02 & $7.21 * *$ & -0.34 & $0.05 * *$ & $34.41^{* *}$ \\
\hline Matar Ageta- 7 x VL-Ageti Matar-7 & 5.57 & $-6.08^{* *}$ & $2.16^{* *}$ & -0.67 & -0.03 & $-6.67 * *$ & $-0.43^{*}$ & $-0.45^{* *}$ & -5.43 \\
\hline Matar Ageta- 7 x Azad Pea-1 & $-6.09 *$ & 0.17 & $-8.4 *$ & -0.33 & 0.01 & -0.54 & $0.77 * *$ & $0.40 * *$ & $-28.99 * *$ \\
\hline Vivek Matar-10 x P-89 & $7.56^{* *}$ & $1.08 * *$ & $0.71^{*}$ & 0.14 & $-0.11^{* *}$ & 1.04 & $-0.78^{* *}$ & $0.24 * *$ & $-19.38 * *$ \\
\hline Vivek Matar-10 x VL-Ageti Matar-7 & $-14.17 * *$ & $7.08 * *$ & $-1.10^{* *}$ & -0.41 & 0.04 & $8.17 * *$ & $0.66^{* *}$ & $-0.33^{* *}$ & -4.93 \\
\hline Vivek Matar-10 x Azad Pea-1 & $6.60 *$ & $-8.17 * *$ & 0.39 & 0.26 & $0.07 *$ & $-9.21^{* *}$ & 0.12 & $0.09 * *$ & $24.31 * *$ \\
\hline Arkel x P-89 & -2.30 & -0.58 & $-1.18 * *$ & -0.29 & 0.02 & $-2.79 * *$ & $-0.54 * *$ & $0.25 * *$ & $-9.88 * *$ \\
\hline Arkel x VL-Ageti Matar-7 & $-16.07 * *$ & $-6.58 * *$ & $0.68 *$ & -0.02 & -0.03 & $-7.17 * *$ & -0.24 & $-0.52 * *$ & -5.23 \\
\hline Arkel x Azad Pea-1 & $18.37 * *$ & $7.17 * *$ & 0.50 & 0.31 & 0.01 & $9.96 * *$ & $0.78^{* *}$ & $0.27 * *$ & $15.13^{* *}$ \\
\hline $\mathbf{S E}(\mathbf{S i})+$ & 2.86 & 0.38 & 0.34 & 0.38 & 0.03 & 0.87 & 0.18 & 0.01 & 3.28 \\
\hline Sij-Skl+ & 4.04 & 0.54 & 0.47 & 0.54 & 0.05 & 1.23 & 0.26 & 0.01 & 4.63 \\
\hline Sij-Sik+ & 8.41 & 1.13 & 0.99 & 1.12 & 0.10 & 2.56 & 0.55 & 0.03 & 9.64 \\
\hline $\mathrm{CD}(\mathrm{p}=0.05)$ & 5.70 & 0.77 & 0.67 & 0.76 & 0.07 & 1.74 & 0.37 & 0.02 & 6.53 \\
\hline $\mathrm{CD}(\mathrm{p}=0.01)$ & 7.56 & 1.02 & 0.89 & 1.01 & 0.09 & 2.31 & 0.49 & 0.03 & 8.68 \\
\hline
\end{tabular}


Table.5 Estimates of specific combining ability effects for various traits in peas (Pisum sativum L.) contd....

\begin{tabular}{|c|c|c|c|c|c|c|c|c|c|}
\hline Hybrids & $\begin{array}{l}\text { Number of } \\
\text { seed per } \\
\text { pod }\end{array}$ & $\begin{array}{l}\text { Number of primary } \\
\text { branches per plant }\end{array}$ & $\begin{array}{c}\text { Shelling } \\
\text { percentage }(\%)\end{array}$ & $\begin{array}{c}\text { Ascorbic Acid } \\
\text { content } \\
(\mathbf{m g} / \mathbf{1 0 0 g})\end{array}$ & $\begin{array}{r}\text { Total Sugar } \\
\text { Content }(\%)\end{array}$ & $\begin{array}{c}\text { Crude Protein } \\
\text { (\%) }\end{array}$ & $\begin{array}{c}\text { Total Soluble } \\
\text { Solids } \\
\left(\text { Brix }=\mathbf{B}^{0}\right)\end{array}$ & $\begin{array}{l}\text { Pod yield per } \\
\text { plant }(\mathrm{g})\end{array}$ & $\begin{array}{l}\text { Pod yield per } \\
\text { hectare }(q)\end{array}$ \\
\hline Palam Priya x P-89 & $-0.87 * *$ & -0.06 & $-2.01 * *$ & $0.17 *$ & 0.24 & $0.74 * *$ & 0.21 & $-4.31 * *$ & $-9.58 * *$ \\
\hline Palam Priya x VL-Ageti Matar-7 & $0.81 * *$ & $0.32 * *$ & $-1.34 *$ & 0.15 & 0.02 & $-0.40 * *$ & 0.10 & 0.53 & 1.17 \\
\hline Palam Priya x Azad Pea-1 & 0.06 & $-0.26^{*}$ & $3.35 * *$ & $-0.32 * *$ & -0.26 & $-0.34^{*}$ & $-0.30 *$ & $3.78 * *$ & $8.41 * *$ \\
\hline Arka Ajit x P-89 & 0.03 & -0.23 & 0.02 & -0.15 & -0.06 & $-0.83 * *$ & 0.05 & $-3.47 *$ & $-7.77 *$ \\
\hline Arka Ajit x VL-Ageti Matar-7 & $-0.29 *$ & $-0.44 * *$ & 0.50 & -0.08 & -0.24 & $1.02 * *$ & 0.06 & 0.70 & 1.69 \\
\hline Arka Ajit x Azad Pea-1 & $0.26^{*}$ & $0.67 * *$ & -0.52 & $0.22 *$ & $0.29 *$ & -0.19 & -0.11 & 2.77 & 6.09 \\
\hline Azad Pea-3 x P-89 & -0.04 & 0.01 & -0.88 & $0.38^{* *}$ & -0.17 & $1.13 * *$ & $0.49 * *$ & $4.48 * *$ & $9.96 * *$ \\
\hline Azad Pea-3 x VL-Ageti Matar-7 & $-0.36 * *$ & $-0.71 * *$ & 0.80 & $-0.29 * *$ & -0.19 & 0.06 & $0.29 *$ & 2.46 & 5.45 \\
\hline Azad Pea-3 x Azad Pea-1 & $0.39 * *$ & $0.71 * *$ & 0.08 & -0.09 & $0.36^{*}$ & $-1.19 * *$ & $-0.77 * *$ & $-6.94 * *$ & $-15.42^{* *}$ \\
\hline Arka Apoorva x P-89 & 0.13 & -0.23 & -0.18 & -0.15 & -0.24 & $-0.79 * *$ & $0.94 * *$ & -1.63 & -3.62 \\
\hline Arka Apoorva x VL-Ageti Matar-7 & $0.9 . * *$ & $0.36 * *$ & -0.40 & $0.37 * *$ & 0.17 & 0.12 & $-1.35^{* *}$ & -0.92 & -2.06 \\
\hline Arka Apoorva x Azad Pea-1 & $-1.04 * *$ & -0.13 & 0.58 & $-0.23 * *$ & 0.07 & $0.67 * *$ & $0.41 * *$ & 2.55 & 5.68 \\
\hline Arka Karthik x P-89 & 0.09 & $-1.13 * *$ & 0.12 & -0.14 & $0.55^{* *}$ & 0.11 & $-1.09 * *$ & 0.17 & 0.38 \\
\hline Arka Karthik x VL-Ageti Matar-7 & -0.52 & $1.96 * *$ & -0.50 & -0.02 & $-0.36^{*}$ & 0.15 & $0.53 * *$ & -1.33 & -2.96 \\
\hline Arka Karthik x Azad Pea-1 & $0.43 * *$ & $-0.83 * *$ & 0.38 & 0.16 & -0.19 & -0.26 & $0.56 * *$ & 1.17 & 2.60 \\
\hline Palam Triloki x P-89 & $-0.37 * *$ & -0.16 & -0.84 & 0.14 & -0.04 & $-1.33 * *$ & $1.17 * *$ & 0.72 & 1.61 \\
\hline Palam Triloki x VL-Ageti Matar-7 & $-0.39 * *$ & 0.22 & 0.33 & -0.12 & $-0.50 * *$ & $0.81 * *$ & $-0.98 * *$ & 1.83 & 4.06 \\
\hline Palam Triloki x Azad Pea-1 & $0.76^{* *}$ & -0.06 & 0.51 & -0.02 & $0.54 * *$ & $0.52 * *$ & -0.19 & -255 & -5.66 \\
\hline Vivek Matar-11 x P-89 & $0.83 * *$ & $-0.26^{*}$ & -0.48 & $0.39 * *$ & $-0.41 * *$ & $-0.42 * *$ & -0.16 & $3.43 *$ & $7.62 *$ \\
\hline Vivek Matar-11 x VL-Ageti Matar-7 & -0.19 & $0.42 * *$ & 0.20 & $-0.30 * *$ & $0.43 * *$ & $0.39 * *$ & -0.23 & $-3.67 *$ & $-8.16 *$ \\
\hline Vivek Matar-11 x Azad Pea-1 & $-0.64 * *$ & -0.16 & 0.28 & -0.09 & -0.01 & 0.03 & $0.39 * *$ & 0.24 & 0.53 \\
\hline Arka Priya x P-89 & -0.14 & $0.71 * *$ & $-1.31 *$ & $-0.20 *$ & $-0.48 * *$ & $-1.52 * *$ & $-0.48 * *$ & 1.93 & 4.29 \\
\hline Arka Priyax VL-Ageti Matar-7 & -0.16 & $-0.41 * *$ & 0.96 & $0.33^{* *}$ & 0.23 & $0.29 *$ & $0.85^{* *}$ & $-4.74 * *$ & $-10.55 * *$ \\
\hline Arka Priya x Azad Pea-1 & $0.29 * *$ & $-0.29 *$ & 0.35 & -0.12 & 0.25 & $1.23 * *$ & $-0.37 * *$ & 2.81 & 6.26 \\
\hline Mithi Phali x P-89 & $1.19 * *$ & $0.61 * *$ & $1.49 * *$ & -0.16 & -0.12 & 0.28 & $0.38 * *$ & -0.66 & -1.46 \\
\hline Mithi Phali x VL-Ageti Matar-7 & $-1.42 * *$ & -0.11 & 0.16 & -0.02 & -0.26 & $-0.91 * *$ & $0.82 * *$ & 0.96 & 2.13 \\
\hline Mithi Phali x Azad Pea-1 & $0.23 *$ & $-0.49 * *$ & $-1.65 * *$ & $0.18 *$ & $0.38^{* *}$ & $0.63 * *$ & $-1.19 * *$ & -0.30 & -0.67 \\
\hline Matar Ageta- 7 x P-89 & $-0.87 * *$ & $-0.53 * *$ & -0.14 & -0.10 & 0.18 & $1.74 * *$ & $-1.02 * *$ & 0.74 & 1.66 \\
\hline Matar Ageta- 7 x VL-Ageti Matar-7 & $0.31 * *$ & -0.94 & 0.13 & -0.09 & -0.09 & $-1.33 * *$ & $0.45^{* *}$ & $3.44^{*}$ & 7.64* \\
\hline Matar Ageta- 7 x Azad Pea-1 & $0.56 * *$ & 1.47 & 0.01 & $0.19 *$ & -0.08 & $--0.42 * *$ & $0.56 * *$ & -4.19 & $-9.30 * *$ \\
\hline Vivek Matar-10 x P-89 & $-0.81 * *$ & $0.61 * *$ & $2.62 * *$ & -0.02 & -0.16 & $0.78 * *$ & $0.47 * *$ & -2.35 & -5.21 \\
\hline Vivek Matar-10 x VL-Ageti Matar-7 & $0.68 * *$ & $-0.51 * *$ & -0.20 & 0.05 & $0.63 * *$ & $-0.32 *$ & $-0.60 * *$ & 1.05 & 2.32 \\
\hline Vivek Matar-10 x Azad Pea-1 & 0.13 & -0.09 & $-2.42 * *$ & -0.03 & -0.47 & -0.46 & 0.13 & 1.30 & 2.89 \\
\hline Arkel x P-89 & $0.83 * *$ & $0.67 * *$ & $-1.59 * *$ & -0.16 & $0.71 * *$ & 0.12 & $-0.94 * *$ & 0.95 & 2.13 \\
\hline Arkel x VL-Ageti Matar-7 & $0.61 * *$ & -0.14 & -0.64 & 0.02 & 0.17 & 0.11 & 0.05 & -0.32 & -0.72 \\
\hline Arkel x Azad Pea-1 & $-1.44 * *$ & $-0.53 * *$ & -0.95 & 0.15 & $-0.88 * *$ & -0.23 & $0.89 * *$ & -0.63 & -1.41 \\
\hline $\mathbf{S E}(\mathbf{S i})+$ & 0.11 & 0.11 & 0.53 & 0.08 & 0.14 & 0.14 & 0.12 & 1.43 & 3.17 \\
\hline Sij-Skl+ & 0.16 & 0.16 & 0.74 & 0.12 & 0.19 & 0.20 & 0.18 & 2.02 & 4.49 \\
\hline Sij-Sik+ & 0.33 & 0.34 & 1.55 & 0.25 & 0.41 & 0.43 & 0.37 & 4.20 & 9.34 \\
\hline CD $(p=0.05)$ & 0.22 & 0.23 & 1.05 & 0.17 & 0.27 & 0.29 & 0.25 & 2.85 & 6.33 \\
\hline $\mathrm{CD}(\mathrm{p}=\mathbf{0 . 0 1})$ & 0.29 & 0.30 & 1.39 & 0.22 & 0.36 & 0.38 & 0.33 & 3.78 & 8.41 \\
\hline
\end{tabular}

*Significant at $5 \%$ level of significance ** Significant at $1 \%$ level of significance 
Table.6 Estimates of General Combining Ability (GCA) effects of testers for various traits in pea (Pisum sativum L.)

\begin{tabular}{|c|c|c|c|c|c|c|c|c|c|}
\hline Male Parent & $\begin{array}{c}\text { Plant } \\
\text { Height } \\
\text { (cm) }\end{array}$ & $\begin{array}{c}\text { Days to } 50 \% \\
\text { flowering }\end{array}$ & $\begin{array}{c}\text { Node at } \\
\text { which first } \\
\text { flower } \\
\text { appears }\end{array}$ & $\begin{array}{c}\text { Inter } \\
\text { nodal } \\
\text { distance } \\
(\mathbf{c m})\end{array}$ & $\begin{array}{c}\text { Number } \\
\text { of pods } \\
\text { per node }\end{array}$ & $\begin{array}{c}\text { Days to } \\
\text { first } \\
\text { picking }\end{array}$ & $\begin{array}{c}\text { Pod } \\
\text { length } \\
\text { (cm) }\end{array}$ & $\begin{array}{c}\text { Pod } \\
\text { width } \\
(\mathrm{cm})\end{array}$ & $\begin{array}{c}\text { Number of } \\
\text { pods per } \\
\text { plant }\end{array}$ \\
\hline P-89 & $-8.38 * *$ & $-0.75 * *$ & -0.01 & $-0.26^{*}$ & $-0.02 *$ & $-0.54 *$ & $0.72 * *$ & $-0.27 * *$ & -0.05 \\
\hline VL Ageti Matar-7 & $4.59 * *$ & $-5.75 * *$ & -0.19 & -0.11 & $0.03 * *$ & $-5.67 * *$ & $-0.50 * *$ & $0.40 * *$ & -1.51 \\
\hline Azad Pea-1 & $3.79 * *$ & $6.5 * *$ & $0.21 *$ & $0.37 * *$ & -0.01 & 6.21 & $-0.21 * *$ & $-0.13 * *$ & 1.55 \\
\hline SE(gi)+ & 0.82 & 0.11 & 0.10 & 0.11 & 0.01 & 0.25 & 0.05 & 0.00 & 0.94 \\
\hline SE(gi-gj)+ & 1.17 & 0.16 & 0.14 & 0.15 & 0.01 & 0.35 & 0.07 & 0.00 & 1.33 \\
\hline CD $(p=0.05)$ & 1.64 & 0.22 & 0.19 & 0.22 & 0.02 & 0.50 & 0.10 & 0.00 & 1.89 \\
\hline$C D(p=0.01)$ & 2.18 & 0.29 & 0.26 & 0.29 & 0.03 & 0.67 & 0.14 & 0.00 & 2.50 \\
\hline
\end{tabular}

*Significant at $5 \%$ level of significance **Significant at $1 \%$ level of significance

Table.6 Estimates of general combining ability effects of testers for various traits in pea (Pisum sativum L.) Conti......

\begin{tabular}{|c|c|c|c|c|c|c|c|c|c|}
\hline Male Parent & $\begin{array}{c}\text { Number of } \\
\text { seed per } \\
\text { pod }\end{array}$ & $\begin{array}{c}\text { Number } \\
\text { of } \\
\text { primary } \\
\text { branches } \\
\text { per plant }\end{array}$ & $\begin{array}{c}\text { Shelling } \\
\text { percentag } \\
\text { e }(\%)\end{array}$ & $\begin{array}{c}\text { Ascorbic } \\
\text { Acid } \\
\text { content } \\
(\mathbf{m g} / \mathbf{1 0 0 g})\end{array}$ & $\begin{array}{l}\text { Total Sugar } \\
\text { Content }(\%)\end{array}$ & $\begin{array}{c}\text { Crude } \\
\text { Protein } \\
(\%)\end{array}$ & $\begin{array}{c}\text { Total } \\
\text { Soluble } \\
\text { Solids } \\
\left(\text { Brix }=\mathbf{B}^{0}\right)\end{array}$ & $\begin{array}{l}\text { Pod yield } \\
\text { per plant } \\
\text { (g) }\end{array}$ & $\begin{array}{c}\text { Pod yield } \\
\text { per hectare } \\
\text { (q) }\end{array}$ \\
\hline P-89 & $0.57 * *$ & $-0.17 * *$ & $1.14 * *$ & $0.08^{* *}$ & $0.42 * *$ & $2.18 * *$ & $0.45^{* *}$ & $13.11 * *$ & $29.13 * *$ \\
\hline VL Ageti Matar-7 & $-0.41 * *$ & -0.056 & $-1.03 * *$ & -0.04 & $-0.28 * *$ & $-0.71 * *$ & $-0.29 * *$ & $-3.48 * *$ & $-7.72 * *$ \\
\hline Azad Pea-1 & $-0.16^{* *}$ & $0.23 * *$ & -0.11 & -0.04 & $-0.14 * *$ & $-1.46 * *$ & $-0.17 * *$ & $-9.6 * *$ & $-21.41 * *$ \\
\hline SE(gi)+ & 0.03 & 0.03 & 0.15 & 0.02 & 0.04 & 0.04 & 0.04 & 0.41 & 0.92 \\
\hline SE $(g i-g j)+$ & 0.04 & 0.05 & 0.21 & 0.03 & 0.06 & 0.06 & 0.05 & 2.01 & 1.30 \\
\hline CD $(p=0.05)$ & 0.06 & 0.07 & 0.30 & 0.05 & 0.08 & 0.08 & 0.07 & 0.82 & 1.83 \\
\hline$C D(p=0.01)$ & 0.08 & 0.09 & 0.40 & 0.06 & 0.10 & 0.11 & 0.09 & 0.09 & 2.43 \\
\hline
\end{tabular}

*Significant at $5 \%$ level of significance **Significant at $1 \%$ level of significance 
Among testers, P-89 was found to be the best general combiner for pod yield per hectare, pod yield per plant, crude protein, total sugar content, total soluble solids; days to $50 \%$ flowering, inter nodal distance, days to first picking, pod length, number of seeds per pod, shelling percentage; VL-Ageti Matar-7 for plant height, days to $50 \%$ flowering, number of pods per node, days to first picking, pod width; Azad Pea-1 for plant height, number of primary branches per plant (Table 5). The results are in agreement with earlier finding of Askander and Osman (2018), Katoch et al., (2017), Kumari et al., (2015), Sharma et al., (2015), Al-Hamdany (2014), Mishra et al., (2014), Mehmed (2013), Punia et al.(2011), Borah (2009), Sharma et al., (2007), Narayan (2006).

It was observed that performance of parents bear direct relation for their respective gca effects in the parents, which showed highest gca effects for different traits were also observed good performance with respect to that particular traits. But this is not true always.

\section{Estimates of Specific Combining Ability effect}

Specific combining ability effects represent dominance and epistatic components of variation, which are non- fixable and can be related with heterosis (Sprague and Tatum, 1942 and Rajas and Sprague, 1952). The best hybrids possessing sca effects in the desired direction for fruit yield and yield components are presented in (Table 6). Negative effects are considered to be desirable for days to 50 per cent flowering (as earliness is preferred over lateness). Specific Combining Ability estimates suggests that the cross Palam Priya $x$ VL-Ageti Matar-7 was found to be the best specific combination for number of seeds per pod, pod length, number of pods per node; Palam Priya x Azad Pea-1 for pod yield per plant, pod yield per hectare, shelling percentage; Arka Ajit x P-89 for days to $50 \%$ flowering, days to first picking, pod width; Arka Ajit x VL-Ageti Matar-7 for crude protein; Arka Ajit x Azad Pea-1 for number of primary branches per plant, ascorbic acid content; Azad Pea-3 x P-89 for ascorbic acid content, crude protein, pod yield per plant, pod yield per hectare; Azad Pea-3 x VL-Ageti Matar-7 for days to $50 \%$ flowering, days to first picking; Azad Pea-3 x Azad Pea-1 for number of pods per plant, number of primary branches per plant; Arka Apoorva x P-89 for total soluble solids; Arka Apoorva x VLAgeti Matar-7 for number of pods per plant, ascorbic acid content; Arka Karthik x P-89 for plant height, crude protein; Arka Karthik x VL-Ageti Matar-7 for number of pods per node, number of primary branches per plant; Palam Triloki x P-89 for plant height, number of pods per node, pod width, total soluble solids; Palam Triloki x VL-Ageti Matar-7 for plant height, node at which first flower appears, crude protein; Palam Triloki x Azad Pea-1 for days to $50 \%$ flowering, days to first picking, number of seeds per pod, total soluble solids; Vivek Matar-11 x P-89 for pod length, pod width, ascorbic acid content, pod yield per plant, pod yield per hectare; Vivek Matar-11 x VL-Ageti Matar-7 for days to $50 \%$ flowering, days to first picking; Arka Priya x P-89 for number of primary branches per plant; Arka Priya x VL-Ageti Matar-7 for pod width, ascorbic acid content, total soluble solids; Arka Priya x Azad Pea-1 for number of pods per node, crude protein, total sugar content; Mithi Phali x P-89 for pod length, number of seeds per pod, shelling percentage; Mithi Phali x VL-Ageti Matar-7 for total soluble solids; Mithi Phali x Azad Pea-1 for plant height; Matar Ageta-7 x P-89 for node at which first flower appears, number of pods per plant, crude protein; Matar Ageta-7 x VLAgeti Matar-7 for pod yield per plant, pod yield per hectare; Matar Ageta-7 x Azad Pea1 for pod length, pod width; Vivek Matar-10 
x P-89 for shelling percentage; Vivek Matar10 x VL-Ageti Matar-7 for node at which first flower appears, number of seeds per pod, total sugar content; Vivek Matar-10 x Azad Pea-1 for days to $50 \%$ flowering, number of pods per node, days to first picking, number of pods per plant; Arkel x P-89 for node at which first flower appears, number of seeds per pod, number of primary branches per plant, total sugar content; Arkel x VL-Ageti Matar-7 for total soluble solids; Arkel x Azad Pea-1 for plant height, pod length, number of pods per plant. The specific combining ability, which represents the predominance of non-additive gene action, is a major component that may be utilized in heterosis breeding. Similar result were observed by Shivaputra et al., (2018), Singh \& Dall (2018), Ram et al., (2018), Katoch et al., (2017), Nageshwar et al., (2017), Chouhan et al., (2016), Sharma et al (2015), Esposito et al., (2014), Singh et al., (2013), Brar et al., (2012) Borah (2009), Sharma et al., (2007) and Narayan (2006).

Heterosis in the cross involving poor $\mathrm{x}$ good combiners might be due to dominant $x$ additive type of interaction, which is partially fixable and the crosses involving both the poor combining parents showing high sca (Sivagama Sundari et al., 1992). The cross Palam Triloki x P-89 show significantly high sca for number of pods per node (Table 6). But both the parents showed negative significant gca effect due to complementation of favorable genes for this trait (Rewale et al., (2003). Therefore it cannot be generalized that the parents with high gca effects can only produce good hybrids. The findings of present investigation revealed that Palam Triloki, Vivek Matar-10, Palam Priya, Azad Pea-3, Arka Karthik and Arka Ajit and testers P-89 and VL-Ageti Matar-7 were best general combiners. Hence, can be used as parents in the hybridization program to get desirable recombinants in segregating generations. The cross Palam Triloki x P-89 could be exploited for number of pods per node. The crosses Palam Priya x VL-Ageti Matar-7, Palam Priya x Azad Pea-1, Arka Ajit x P-89, Arka Ajit x VL-Ageti Matar-7, Arka Ajit x Azad Pea-1 and Azad Pea-3 x P-89 showed maximum sca effects for most of the traits studied including the yield and yield attributing traits. Thus, these crosses could be advanced to recover desirable segrega for the improvement of yield and yield contributing traits Result showed that non additive gene action is an integral component of the genetic architecture of different characters in the material used in pea.

\section{References}

Al-Hamdany, H. Sh.Y. 2014. Estimation of heterosis, combining ability, gene action, genotypic and phenotypic correlation in pea (Pisum sativum L.). Jordan J. of Agri. Sci., 10 (2).

Anonymous 2017-18. Annual report of area and production of vegetables. NHB database, Gurgoan, India. NHB. http://www.nhb.gov.in.

Askander, H.S. and Osman, K.F. 2018. Heterosis and combining ability effects for some traits of pea (Pisum sativum L.). Mesopotamia J. of Agric 46(4): 435-449.

Borah, H. K. 2009. Study on combining ability and heterosis in field pea. Agri. Res. Comm. Centre, Leg. Res., 32(4): 255-259.

Brar, P. S., R. K. Dhall and Dinesh, 2012. Heterosis and combining ability in field pea for yield and its component. Vegetable Science, 39(1): 51-54.

Chauhan, S., Ravindrababu, Y., Patel, A. and Mishra S. 2016. Heterosis, combining ability and molecular characterization in fieldpea (Pisum sativum L. var arvense). J. Food Legumes., 29 (1): $18-21$. 
Espósito, M.A., Gatti, I., Cravero, V.P., Anido, A.F. and Cointry, L.E. 2014. Combining abilities and heterotic groups in pea. AJCS, 7(11):1634-1641.

Kempthorne, O. 1957. An introduction to Genetics statistics. John Wiley and Sons, Inc New York, pp 458-71.

Katoch, V., Bharti, A., Sharma, A., Rathore, N. and Kumri, V. 2017. Heterosis and combining ability studies for economic traits in garden pea (Pisum sativum L.). Legume Research LR-3849 (1-8).

Kumari, J. Dikshit, H.K., Singh, B. and Singh, D. 2015. Combining ability and character association of agronomic and biochemical traits in pea (Pisum sativum L.). Scientia Horticulturae, 181:26-33.

Mehmed A, 2013. Determination of some agricultural characters of pea (Pisum sativum L.). J. Animal and Veterinary Adv., 12(7): 798-802.

Mishra, V.D.,Singh, H., Singh, P.K., Lal, G.M., Prasad, R.D. and Singh, S.K. 2014. Study on combining ability effects for seed yield and its component characters in field pea (Pisum sativum L.). Annals of Agri-Bio Research 19(4): 728-732.

Nageshwar, Kumar, B., Suman, H., Madakemohekar, A.H. and Tamatam, D. 2017. Combining ability and heterosis analysis for grain yield and yield associated traits in pea (Pisum sativum L.). Legume Research. LR3955:1-7.

Narayan, R. 2006. Combining ability for quality attributes in garden pea. Environment and ecology (Special 2), Pp. 464-467.

Punia, S.S., Ram, B., Verma, P., Koli, N.R. and Rokaria, P. 2011. Combining ability studies in field pea (Pisum sativum L.). J. of food Legumes 24(3): 242-244.

Ram, H., Dhar, S., Choudhary, H., Sharma,
R.K., Solanke, A.U. Prasad, L. and Tomar, B. S. 2018. Combining ability analysis for earliness and yield related characters in pea (Pisum sativum L.). The Indian Journal of Agricultural Sciences, 88(11): 22-26.

Rajas, B. A. and G. F. Sprague. 1952. A comparison of variance components in corn yield traits III. General and specific combining ability and their interaction with location and years. Agron J., 46: 462-466.

Rewale, V. S., Bendale, V.W., Bhave, S. G., Madav, R.R. and B.B. Jadhav 2003. Heterosis for Yield and Yield components in okra. J. Maharashtra agric. Univ., 28 (3): 247-249.

Sharma, A., Singh, G., Sharma, S., Sood, S., 2007. Combining ability and heterosis for pod yield and its related horticultural traits in garden pea (Pisum sativum L.)s under mid-hill sub temperate and high hill dry temperate conditions of Himachal Pradesh. Indian J. Genet. 67(1): 47-50.

Sharma, B. B., V. K. Sharma., M. K. Dhakar and S. Punetha, 2014. Combining ability and gene action studies for horticultural traits in garden pea. Inter. J. Fruit and Veg. Sci., 1 (1): 007-13.

Sharma, B.B., Sharma, V.K. and Dhar, S. 2015. Combining ability studies for yield and other horticultural traits in garden pea (Pisum sativum var. hortense L.). Indian J. Horti. 72(2):500-505

Shivaputra, Kale, V. S. Meghwal, M.L. and Kumar, H. 2018. Combining Ability studies in garden pea (Pisum sativa Var. Hortense). Int. J. Curr. Microbiol. App. Sci. 6: 785-790.

Singh and Dhall. 2018. Heterotic potential and combining ability of yield and quality traits in garden pea (Pisum sativum L.). Vegetable Science, 45(1): 7-13 
Singh, V., Singh, R.J., and Chauhan, M.P. 2013. Combining ability analysis for some agronomically important traits in pea under disease protected and unprotected conditions. Legume Res., 36 (3): 200-207.

Sivagamasundhari, S., Irulappan, I., Arumugam, R. and Jayasankar, S.,
1992. Combining ability in okra (Abelmoschus esculentus L.Moench). South Indian Hort., 40:21-27.

Sprague, G. F. and L. A.s Tatum, 1942. General vs specific combining ability in single crosses of corn. J. Amer. Soc. Agron., 34: 923-932.

\section{How to cite this article:}

Manoj Kumar, R. K. Gupta, Sanjeev Kumar, R. K. Samnotra, Anil Bhushan and Tuhina Dey. 2020. Combining Ability Studies for Various Horticultural Traits in Garden Pea (Pisum sativum L.). Int.J.Curr.Microbiol.App.Sci. 9(12): 841-853.

doi: https://doi.org/10.20546/ijcmas.2020.912.101 